\title{
Finding The Finger By The Boundary Vector Product
}

\author{
Yuan-Fei Cheng \\ Department of Information Management \\ Meiho Institute of Technology, Nei Pu, Ping Tung, Taiwan \\ x2070@email.meiho.edu.tw
}

\begin{abstract}
A finger finding procedure has been presented to recognize the finger number of the hand gesture. The hand gesture is extracted from the stationary background and transformed into a binary image. The hue attribute, that is, the I value in the YIQ color space is used to extract the hand gesture shape. This attribute is not so sensitive to the light change and the other noises while catching the image frames. The binary image of the hand gesture shape is processed with erosion several times to eliminate the noises and then processed with dilation several times to recover the original shape. After the removal of the image noises, a boundary detection algorithm is used to trace the border of the hand shape from the binary image. The border of the hand shape can be used to calculate the vector product along the boundary. From the vector product along the boundary, the finger number of the hand gesture can be obtained.
\end{abstract}

Keywords: finger calculation, hand gesture, boundary detection, vector product

\section{Introduction}

Hand motion analysis has many applications in human machine interface. The hand gesture identification algorithms can be classified into the gloves-based and vision-based methods, and the vision-based methods consists of contour-based and model-based methods [1]. The vision-based methods provide a natural interaction, but such methods have occlusion, noise, and spurious data. The contour-based methods extract the silhouette or contour feature from the hand gesture to provide the needed information. A feature extraction algorithm has been used to extract the hand shape from complex background, which combines the motion, skin color, and edge detections [2]. A similar algorithm will be used to trace the border of the hand shape from the stationary background.

The hand gesture is usually extracted from the stationary background and transformed into a binary image. The RGB color space is usually used in this kind of problems. The skin color information is obtained first, and then used to exact the hand gesture from the background. In this paper, the YIQ color space is used instead of the RGB color space. In the development of the color television system in the United States, NTSC formulated a color coordinate system for transmission composed of three values, Y, I, $\mathrm{Q}$ [3]. The $\mathrm{Y}$ value, called luma, is proportional to the gamma-corrected luminance of a color. The other two components, I and Q, is called chroma and jointly describe the hue and saturation attributes of an image [4]. According to the preliminary test, the I value, that is, the hue attribute of an image can have a best result while extracting the hand gesture form the complex background. This attribute is not so sensitive to the light change and the other noises while catching the image frames.

Although the hue attribute of the image can be used to reduce the noises, the morphological operation is still needed to filter the noises. The binary image of the hand gesture shape is processed with erosion several times to eliminate the noises and then processed with dilation several times to recover the original shape. There are many morphological algorithm can be used [4]. A similar algorithm is used to eliminate the noises quickly in this paper.

There are many boundary detection algorithms can be used to describe the hand shape [5]. A similar algorithm will be used to trace the border of the hand shape from the binary image. The border of hand shape can be used to calculate the vector product along the boundary. From the vector product along the boundary, the finger number of the hand gesture can be obtained. In the following, the whole finger finding procedure is reported.

\section{Hand Shape Extraction}

The hand gesture is extracted from the stationary background and transformed into a binary image. The YIQ color space is used instead of the RGB color space. The I value, that is, the hue attribute of an image in the YIQ color space, can have a best result when extract the hand gesture from the complex 
background. The binary images of the hand gesture shape obtained from different color space are shown in Figure 1. According to the observation, the I value is not so sensitive to the light change and the other noises while catching the image frames. The difference of this attribute between the stationary background and the hand gesture is used to construct the binary image of the hand gesture shape in this paper.

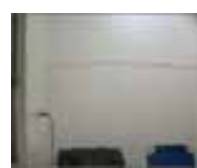

Background

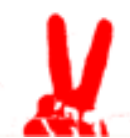

G-color space

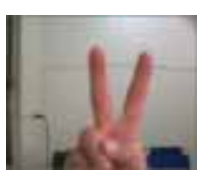

Original

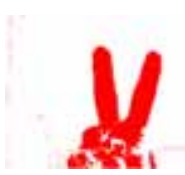

B-color space

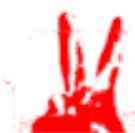

R-color space

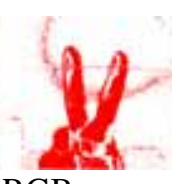

RGB-average

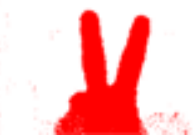

I-color space

Fig. 1: The different binary images.

\section{Morphological Image Processing}

After obtaining the binary image of the hand gesture shape, a morphological operation will be used to filter the noises. The binary image of the hand gesture shape is processed with erosion several times to eliminate the noises and then processed with dilation several times to recover the original shape. A $2 * 2$ pixel pattern is used and the algorithm is shown in Figure 2. All black pixels will be erased if there exists a white pixel during erosion operation, and all black pixels will be recovered if there exists a black pixel during dilation operation. The result after 2 times erosion and 2 times dilation is shown in Figure 3. Most of the noises have been removed in the binary image.

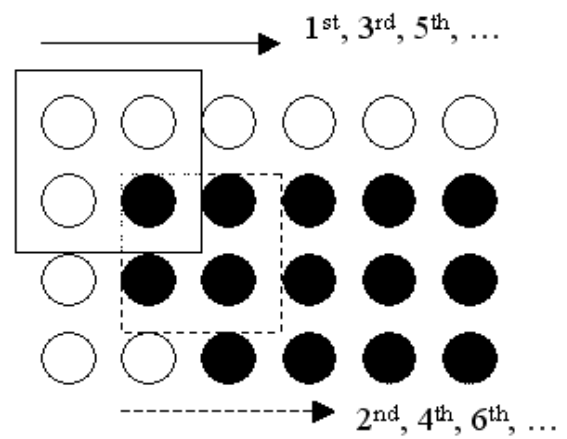

Fig. 2: Morphological operation.

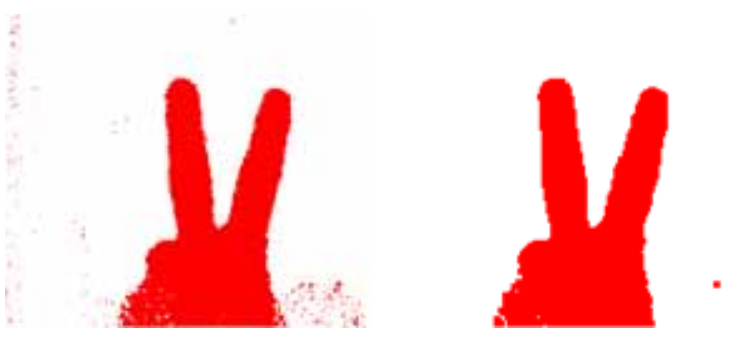

Fig. 3: After erosion and dilation.

\section{Boundary Detection}

The algorithm used for the boundary detection of the binary image is shown in Figure 4. Only one hand shape appeared in the binary image is assumed, and the hand gesture is from the bottom side of the image is also assumed, so that the detection can be started to find the boundary from the right side of the image bottom. The first boundary pixel is chosen about several pixels from the image bottom to avoid the noises in the image boundary, and then move counterclockwise along the border to trace the whole boundary.

In order to find the next boundary pixel, eightconnected pixels are considered. The right connectedpixel is detected first and then detects the next pixel counterclockwise. The first pixel appeared after white pixel is the boundary pixel. This procedure is done recursively until the whole boundary of the hand shape is found. However, if the shape is connected for only one pixel, this procedure may find a previously detected pixel. In this case, the next qualified pixel will be selected. Otherwise, the infinite detection loops will happen. 


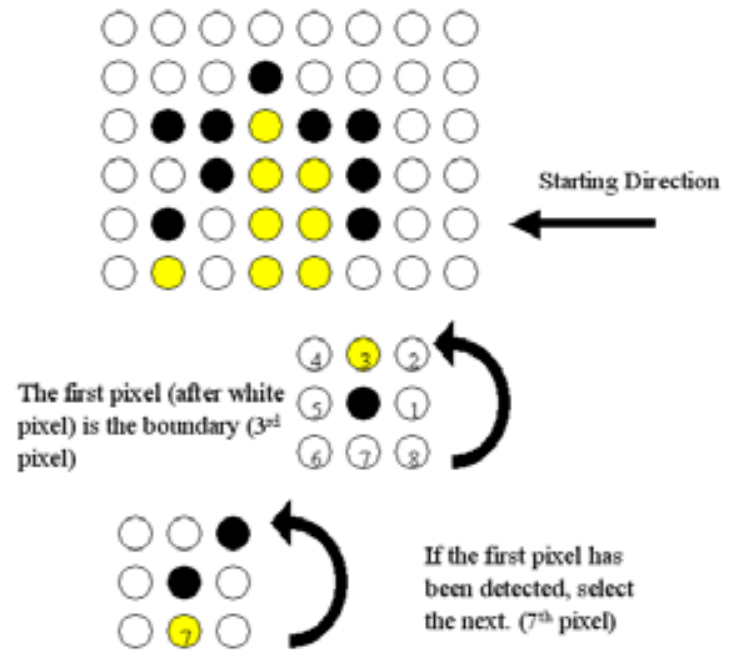

Fig. 4: Boundary detection algorithm.

\section{Vector Product}

The border of the hand shape can be used to calculate the vector product along the boundary. The vector product is calculated counterclockwise as shown in Figure 5. From the vector product along the boundary, some convex points can be found. They are the candidates of the fingers. From the convex points, the inner product can be calculated as shown in Figure 6. If the inner product is below some limit, the convex point can be concluded as the tip of the finger, and the finger number can be calculated.

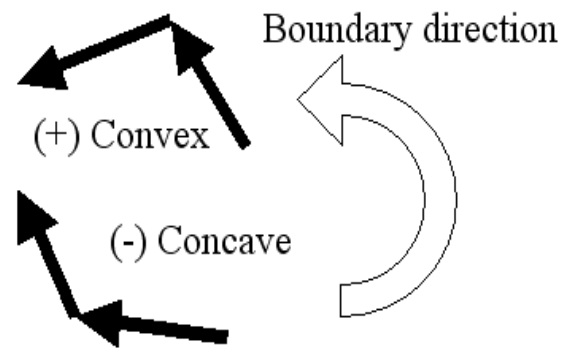

Fig. 5: Vector product.

\section{Inner Product of Convex Point}

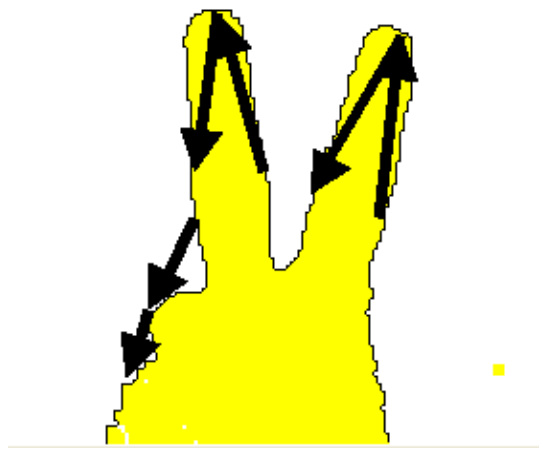

Fig. 6: Verification of finger.

\section{Experiments}

The results using the finger finding procedure are shown in Figure 7. The finger number of the hand gesture can be obtained from the vector product along the border of the hand shape. Some parameters will be used to justify the finger number. These parameters are the vector product interval along the boundary, the positive vector product limit, the negative vector product limit, the inner product interval, and the negative inner product limit.

The vector product interval along the boundary is used to obtain the vector product diagrams in Figure 7. It is 20 in this case. That is, the vector is obtained for every 20 boundary-pixels along the boundary. The positive vector product limit and the negative vector product limit are used to obtain the maximum value of the vector product for every peak. They are 0.7 and 0.2 in this case. That is, only the tips of the peaks higher than 0.7 are considered and each peak ends at the value of -0.2 . The tips are the maximum value for every peak. The considered tips of the peaks are the candidates of the tips of the fingers. The inner product interval is used to obtain the two inner product vectors in the considered tip. It is 60 in this case. That is, the vectors are obtained in the considered tip for every 60 boundary-pixels. If the value of the inner product in the considered tip is below the negative inner product limit, the considered tip is classified as the tip of the finger. Otherwise, it is only the convex point in this hand shape. The smaller circles in Figure 7 are the values of the inner product in the considered tips and the bigger circles in Figure 7 are the qualified tips for every peak. That is, the qualified tips are classified as the tips of the fingers and the finger number can be obtained. 

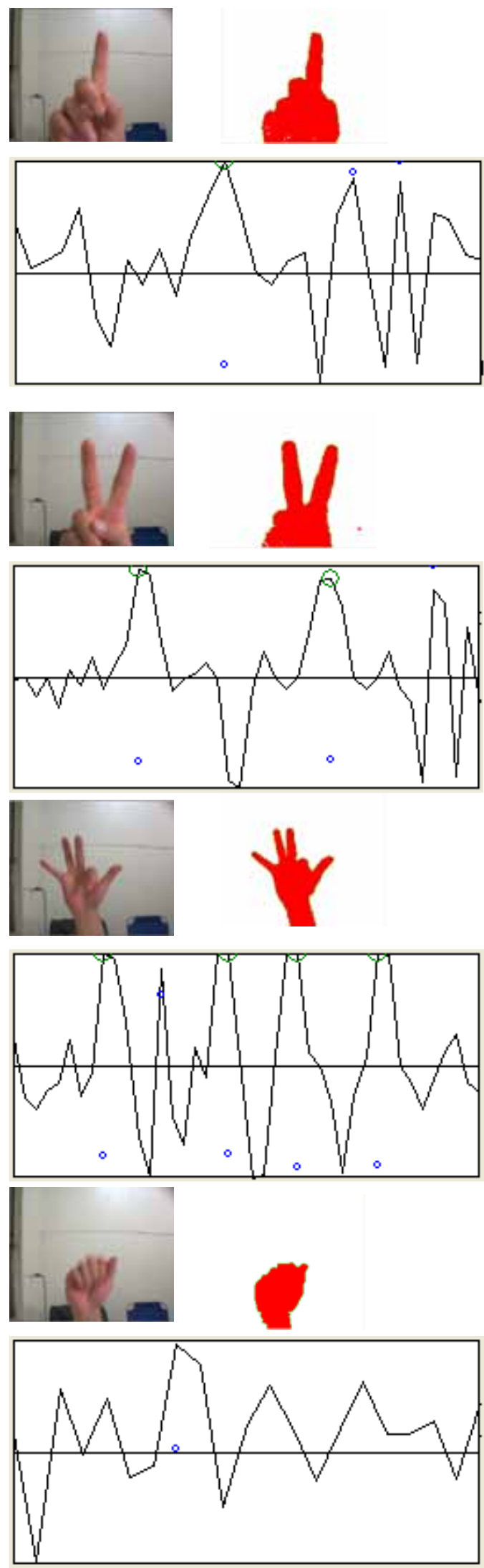

Fig. 7: The vector product along the boundary.

\section{Conclusions}

A finger finding procedure has been presented to recognize the finger number of the hand gesture. The hand gesture is extracted from the stationary background and the border of the hand shape is found using the presented algorithm. The results using the RGB color space and the YIQ color space are compared. The hue attribute in the YIQ color space has the best result. The binary image is processed with erosion 2 times to eliminate the noises and then processed with dilation 2 times to recover the original shape in the experiments. After the removal of the image noises, a boundary detection algorithm is used to trace the border of the hand shape. The border of the hand shape can be used to calculate the vector product along the boundary and the inner product in the convex points. These values are use to verify the finger tips in the hand shape. Some testing results are presented and the whole procedure provides a very good method in calculating the number of the fingers in the hand shape.

\section{References}

[1] Cheng-Chang Lien and Chung-Lin Huang, "Model-based articulated hand motion tracking for gesture recognition”, Image and Vision Computing, Vol. 16, pp. 121-134, 1998

[2] Feng-Sheng Chen, Chih-Ming Fu, and ChungLin Huang, "Hand gesture recognition using a real-time tracking method and hidden Markov models”, Image and Vision Computing, Vol. 21, pp. 745-758, 2003

[3] F. J. Bingley, "Color Vision and Colorimetry", Television Engineering Handbook, D. G. Fink, ed., McGraw-Hill, USA, 1957

[4] William K. Pratt, "Digital Image Processing", third edition, John Wiley \& Sons, Inc, USA, pp. 80-81, pp.401-410, 2001

[5] Milan Sonka, Vaclav Hlavac and Roger Boyle, "Image Processing, Analysis, and Machine Vision”, second edition, Brooks/Cole Publishing Company, USA, pp. 142-148, 1999 